\title{
Increased expression of high mobility group box protein 1 and vascular endothelial growth factor in placenta previa
}

\author{
HAN XIE $^{1 *}$, PING QIAO $^{1 *}$, YI LU $^{1}$, YING LI $^{2}$, YUPING TANG $^{1}$, \\ YIYING HUANG $^{1}$, YIRONG BAO ${ }^{1}$ and $\mathrm{HAO} \mathrm{YING}^{1 *}$ \\ Departments of ${ }^{1}$ Obstetrics and ${ }^{2}$ Pathology, Shanghai First Maternity and Infant Hospital, \\ Tongji University School of Medicine, Shanghai 201204, P.R. China
}

Received May 7, 2016; Accepted March 27, 2017

DOI: $10.3892 / \mathrm{mmr} .2017 .7682$

\begin{abstract}
Placenta previa is often associated with preterm delivery, reduced birth weight, a higher frequency of placental accreta and postpartum haemorrhage, and increased likelihood of blood transfusion. The present study aimed to examine the expression of high mobility group box protein 1 (HMGB1) in the placenta of women with or without placenta previa. The study group consisted of placental tissues obtained from women with or without placenta previa. The expression levels of HMGB1 and vascular endothelial growth factor (VEGF) were evaluated in the placental tissues using reverse transcription-quantitative polymerase chain reaction, western blotting and immunohistochemistry. The mRNA expression levels of HMGB1 and VEGF were significantly increased in the placenta previa group compared with in the normal group. In addition, the placenta previa group exhibited increased HMGB1 and VEGF staining in vascular endothelial cells and trophoblasts. There were no significant differences in the expression of HMGB1 or VEGF between groups with or without placenta accreta or postpartum haemorrhage. The present study hypothesised that the increased expression of HMGB1 in the placenta may be associated with the pathogenesis of placenta previa by regulating the expression of the proangiogenic factor VEGF.
\end{abstract}

\section{Introduction}

Placenta previa is a complication that occurs during pregnancy in which the placenta is partially or wholly attached to the

Correspondence to: Dr Hao Ying, Department of Obstetrics, Shanghai First Maternity and Infant Hospital, Tongji University School of Medicine, 2699 Gaoke West Road, Pudong New Area, Shanghai 201204, P.R. China

E-mail: stephenying_2011@163.com

${ }^{*}$ Contributed equally

Key words: angiogenesis, high mobility group box protein 1, placenta previa, vascular endothelial growth factor lower uterine segment (1). Placenta previa is a leading cause of antepartum haemorrhage. Due to the rising incidence of caesarean section births, along side increasing maternal age, the number of cases of placenta previa, and its complications, which include placenta accreta and postpartum haemorrhage, are expected to increase (2). Placenta accreta is an obstetric complication in which the placental trophoblast invades the endometrium beyond Nitabuch's layer, as a result of a defect in the decidua basalis (3). If the placenta attaches itself even more deeply into the muscle wall of uterus, it will lead to placenta increta. The major morbidity associated with this abnormal placentation is caused by the significant blood loss that occurs during labour, resulting in a longer maternal hospital stay and blood transfusion treatment (4). Risk factors for placenta previa include previous caesarean section delivery, grand multiparity and recurrent miscarriage (5). Although the risk factors for placenta previa are well defined, much less is known regarding its aetiology and underlying molecular mechanisms.

Damage to the endometrium or myometrial uterine lining during abortion or cesarean delivery may significantly damage the endometrium and lead to inflammation, which increases the risk for placenta previa (6). In addition to the classic theory of inflammatory injury, abnormal angiogenesis has recently been considered a novel mechanism underlying placenta previa (7). Data derived from animal and human studies demonstrate that various regulatory molecules serve functional roles in controlling trophoblast invasion and placental angiogenesis (8). As an inflammatory cytokine, the effects of high mobility group box protein 1 (HMGB1) on inflammation, tumourigenesis (9) and pregnancy (10) have been defined. Throughout gestation growth factors such as vascular endothelial growth factor (VEGF) are abundantly secreted from diverse cell types of the fetal-maternal interface and were shown to promote proliferation, adhesion and/or invasion (11). The present study aimed to investigate the expression levels of HMGB1 in the placenta, in order to explore the possible mechanisms underlying placenta previa.

\section{Materials and methods}

Study population. The present study was conducted between November 2014 and January 2015. A total of 37 women without other medical and surgical diseases were recruited to 
the present study at the Shanghai First Maternity and Infant Hospital, Tongji University School of Medicine (Shanghai, China). A total of 22 women had normal term pregnancies, whereas 15 women suffered from placenta previa. In the placenta previa group, 7 women also suffered from placenta accreta and 9 suffered from postpartum haemorrhage. All of the 37 women underwent elective caesarean section. There were no significant differences in age between women in the healthy and placenta previa groups. Written informed consent was obtained from all patients. All experiments, including any relevant details, were approved in advance by the Ethics Committee of the Shanghai First Maternity and Infant Hospital, Tongji University School of Medicine, and were performed in accordance with relevant guidelines and regulations.

Reverse transcription-quantitative polymerase chain reaction $(R T-q P C R)$. As soon as the placenta peeled from the uterine in the caesarean section, samples were harvested from the parental placenta around the umbilical cordunder sterile conditions, rinsed by sterile saline, wiped with a gauze, treated with liquid nitrogen and stored in $80^{\circ} \mathrm{C}$. Total RNA was extracted using TRIzol reagent (Invitrogen; Thermo Fisher Scientific, Inc., Waltham, MA, USA) and cDNA was synthesised from total RNA using the mRNA 1st strand cDNA synthesis kit (BioTNT, Inc., Shanghai, China), according to the manufacturer's protocol. Template cDNA was subjected to PCR amplification using gene-specific sense and antisense primers, which were all designed by BioTNT via Primer 5.0 software, and synthetized by Invitrogen; Thermo Fisher Scientific, Inc. (Table I). Reactions contained $1 \mu \mathrm{l}$ cDNA template, $2 \mu 1$ forward primers, $2 \mu 1$ reverse primer; and $10 \mu \mathrm{l}$ PCR Premix (BioTNT, Inc.) in a total volume of $20 \mu 1$. RT-qPCR thermocycling conditions were as follows: Initial denaturation at $95^{\circ} \mathrm{C}$ for $5 \mathrm{~min}$, followed by 40 cycles of denaturation at $95^{\circ} \mathrm{C}$ for $5 \mathrm{sec}$, annealing and extension at $60^{\circ} \mathrm{C}$ for $30 \mathrm{sec}$. The expression levels of each gene were standardised against the housekeeping gene $\beta$-actin. mRNA expression levels were expressed as a ratio, using the $2^{-\Delta \Delta \mathrm{Cq}}$ method for comparing the relative expression results (12).

Western blot analysis. Frozen samples were homogenized in liquid nitrogen. Protein extracts were prepared by 1:1 dilution of the initial homogenate with radioimmunoprecipitation assay buffer (Wuhan Boster Biological Technology, Ltd., Wuhan, China) in the presence of protease inhibitors and extracted by ultracentrifugation. The bicinchoninic acid assay (Pierce; Thermo Fisher Scientific, Inc.) was used for protein quantitation. A total of $20 \mu \mathrm{g}$ protein for each sample was loaded and separated by $10 \%$ sodium dodecyl sulphate-polyacrylamide gel electrophoresis, and were subsequently transferred to polyvinylidene fluoride membranes (EMD Millipore, Billerica, MA, USA) in blotting buffer (25 $\mathrm{mM}$ Tris, $150 \mathrm{mM}$ glycine and $20 \%$ methanol) for $2 \mathrm{~h}$ at room temperature. After blocking with 5\% skimmed milk in PBS for $1 \mathrm{~h}$ at room temperature, the membranes were then incubated with anti-human HMGB1 rabbit immunoglobulin (Ig)G antibody (catalog no. ab79823; 1:1,000; Abcam, Cambridge, MA, USA) or anti-human (VEGF) rabbit IgG antibody (catalog no. ab46154; 1:1,000; Abcam) at $4^{\circ} \mathrm{C}$ overnight. Anti- $\beta$-actin (catalog no. 4970; 1:3,000; Cell Signaling Technology, Inc., Danvers, MA, USA) was used as an internal control. The membranes were further incubated for $1 \mathrm{~h}$ at room temperature with peroxidase-labelled secondary antibodies (catalog no. sc-2004; 1:1,000; Santa Cruz Biotechnology, Inc., Dallas, TX, USA). The bands were detected using an enhanced chemiluminescence system (Pierce; Thermo Fisher Scientific, Inc.). The protein bands for HMGB1 or VEGF were semi-quantified and normalised to the control band using ImageQuant LAS 4000 (GE Healthcare Life Sciences, Chalfont, UK).

Immunohistochemistry. Placental tissues were fixed with $10 \%$ formalin for $24 \mathrm{~h}$, embedded with paraffin and sliced continuously to sections of $4 \mathrm{~mm}$. Slides were preheated at $70^{\circ} \mathrm{Cfor} 1 \mathrm{~h}$ prior to deparaffinization and rehydration with ethanol. Antigen retrieval was performed in citrate buffer at $95^{\circ} \mathrm{C}$ for $15 \mathrm{~min}$. For detection of HMGB1 expression, the slides were incubated with anti-HMGB1 antibody-ChIP Grade (catalog no. ab18256, 1:100; Abcam) in 5\% bovine serum albumin (Sigma-Aldrich, Merck KGaA, Darmstadt, Germany) for $45 \mathrm{~min}$ at room temperature, and were then incubated with horseradish peroxidase-coupled to streptavidin-conjugated secondary antibody (catalog no. sc-2004; 1:1,000; Santa Cruz Biotechnology, Inc.) for $30 \mathrm{~min}$ at room temperature. Slides stained without the primary antibody were used as negative controls. The resulting signal was developed with diaminobenzidine (Sigma-Aldrich, Merck $\mathrm{KGaA}$ ), according to the manufacturer's protocol, and the sections were counter stained with Mayer's haematoxylin. Staining for VEGF (catalog no. ab46154; 1:1,000; Abcam) was performed using the some staining protocol.

Evaluation of immunohistochemistry. The evaluated sections spanned the whole placental tissue, from the fetal membranes to the decidual plate. Trophoblasts, mesenchymal cells, and villous vascular endothelial cells from the stem villous, mature intermediate villous and terminal villous, were evaluated.

A semi-quantitative scale was used to evaluate HMGB1 and VEGF staining, as follows: Stain intensity was scored between 0 and 3 ( 0 , no staining; 1 , weak but detectable; 2 , moderate or distinct; 3 , intense) and was multiplied by the average percentage of positive staining, which was scored between 0 and 4 ( 0 , no positive cells; $1,0-25 \%$ positive cells; 2, 26-50\% positive cells; $3,51-75 \%$ positive cells; 4 , $76-100 \%$ positive cells) $(13,14)$. In each slide, five areas were evaluated under a microscope (x400 original magnification). Evaluation of immunohistochemistry was performed blindly by three independent investigators and the average score was used. The results were assessed by a pathologist under a light microscope. The agreement between the different investigators was $>90 \%$.

Statistical analysis. Each experiment was repeated three times independently. Results from each independent experiment were expressed as the mean \pm standard deviation. Analysis of enumeration data was performed using Pearson $\chi^{2}$ test. Measurement data were assessed by independent-samples t-test. Statistical analysis of immunohistochemistry results was performed with the Mann-Whitney U-test. Statistical analyses were conducted using SPSS 19.0 statistical software (IBM SPSS, Armonk, NY, USA). P<0.05 was considered to indicate a statistically significant difference. 
Table I. Polymerase chain reaction primers used in the present study.

\begin{tabular}{lll}
\hline Gene & \multicolumn{1}{c}{ Forward (5'-3') } & Reverse $\left(5^{\prime}-3^{\prime}\right)$ \\
\hline HMGB1 & CTGGGAGAGATGTGGAAT A & GCAGCAATATCCTTTTCGT \\
VEGF-A & TGTGCCCACTGAGGAGTC & CATTTGTTGTGCTGTAGGA \\
$\beta$-actin & AAGGTGACAGCA GTCGGT T & TGTGTGGACTTGGGAGAGG
\end{tabular}

HMGB1, high mobility group box protein 1; VEGF, vascular endothelial growth factor.

Table II. Demographic characteristics and outcome data.

\begin{tabular}{|c|c|c|c|}
\hline Variable & Placenta previa $(n=15)$ & Controls $(n=22)$ & P-value \\
\hline \multicolumn{4}{|l|}{ Characteristics at enrolment } \\
\hline Maternal age, years & $30.87 \pm 2.16$ & $30.77 \pm 1.99$ & 0.9340 \\
\hline Gestational age, weeks & $37 \pm 1.0$ & $39 \pm 0.6$ & $<0.0001$ \\
\hline Abortion history, n (\%) & $10(66.7)$ & $5(22.7)$ & 0.0160 \\
\hline Prior caesarean section, $\mathrm{n}(\%)$ & $2(13.3)$ & 0 & 0.0780 \\
\hline \multicolumn{4}{|l|}{ Outcome characteristics } \\
\hline Placenta accreta, n (\%) & $7(46.7)$ & $0(0)$ & 0.0010 \\
\hline Birth weight, $g$ & $3,030 \pm 388$ & $3,413 \pm 260$ & 0.0100 \\
\hline Postpartum haemorrhage, $\mathrm{ml}$ & $902 \pm 1,194$ & $302 \pm 11$ & 0.0230 \\
\hline Blood transfusion, n (\%) & $3(12.5)$ & $0(0)$ & 0.0290 \\
\hline Caesarean hysterectomy, n (\%) & $0(0)$ & $0(0)$ & $\mathrm{N} / \mathrm{A}$ \\
\hline
\end{tabular}

\section{Results}

Clinical characteristics of the enrolled women. The demographic, clinical and pregnancy outcome characteristics of 37 women are presented in Table II. Women with placenta previa had a significantly increased history of abortion and caesarean section births, both of which are known risk factors for placenta previa. Compared with the control group, women in the placenta previa group delivered at an earlier gestational age and gave birth to babies of a lower birth weight. Women with placenta previa had a higher frequency of placental accrete, thus resulting in more cases of postpartum haemorrhage and blood transfusion.

mRNA expression levels of HMGB1 and VEGF. The mRNA expression levels of HMGB1 and VEGF were analysed by RT-qPCR. The mRNA expression levels of HMGB1 and VEGF in the placenta previa group were significantly higher compared with in the normal group (Fig. 1). In the placenta previa group, there were no significant differences in HMGB1 and VEGF mRNA expression between groups with or without placenta accreta, or between groups with or without postpartum haemorrhage.

Protein expression levels of HMGB1 and VEGF. Protein expression was measured by western blotting. The protein expression levels of HMGB1 in the placenta previa group were significantly increased compared with in the normal group (Fig. 2). These findings were similar to the results of the RT-qPCR analysis. In addition, VEGF protein expression levels were higher in the placenta previa group compared with in the normal group; however, this finding was not statistically significant. In the placenta previa group, there were no significant differences in the protein expression levels of HMGB1 and VEGF between groups with or without placenta accreta, or between groups with or without postpartum haemorrhage.

Histological and immunohistochemical examination of HMGB1 in placental tissue. HMGB1 and VEGF protein expression was detected in placental tissue using immunohistochemistry (Fig. 3). The present study detected strong nuclear expression of HMGB1 in vascular endothelial cells and mesenchymal cells from the placenta of women who had normal pregnancies. Immunohistochemical staining also revealed that HMGB1 was slightly expressed in the placental syncytiotrophoblast and cytotrophoblastnuclei. In the placenta previa group homogeneous cytoplasmic expression of HMGB1 was detected in vascular endothelial cells and mesenchymal cells (Fig. 3A). Furthermore, in the placenta previa group increased HMGB1 staining was observed in vascular endothelial cells covering the peripheral chorionic villi, compared with in the normal group. In mesenchymal cells, there was a significant trend toward a higher mean staining intensity in the placenta previa group. The HMGB1 staining score also differed in trophoblast cells between the placenta previa and normal groups. To investigate if HMGB1 is associated with the depth of placental invasion and postpartum haemorrhage, the present study divided the placenta previa group into placenta accreta and control groups. There was no significant difference between the placenta accreta and control groups. Similarly, 

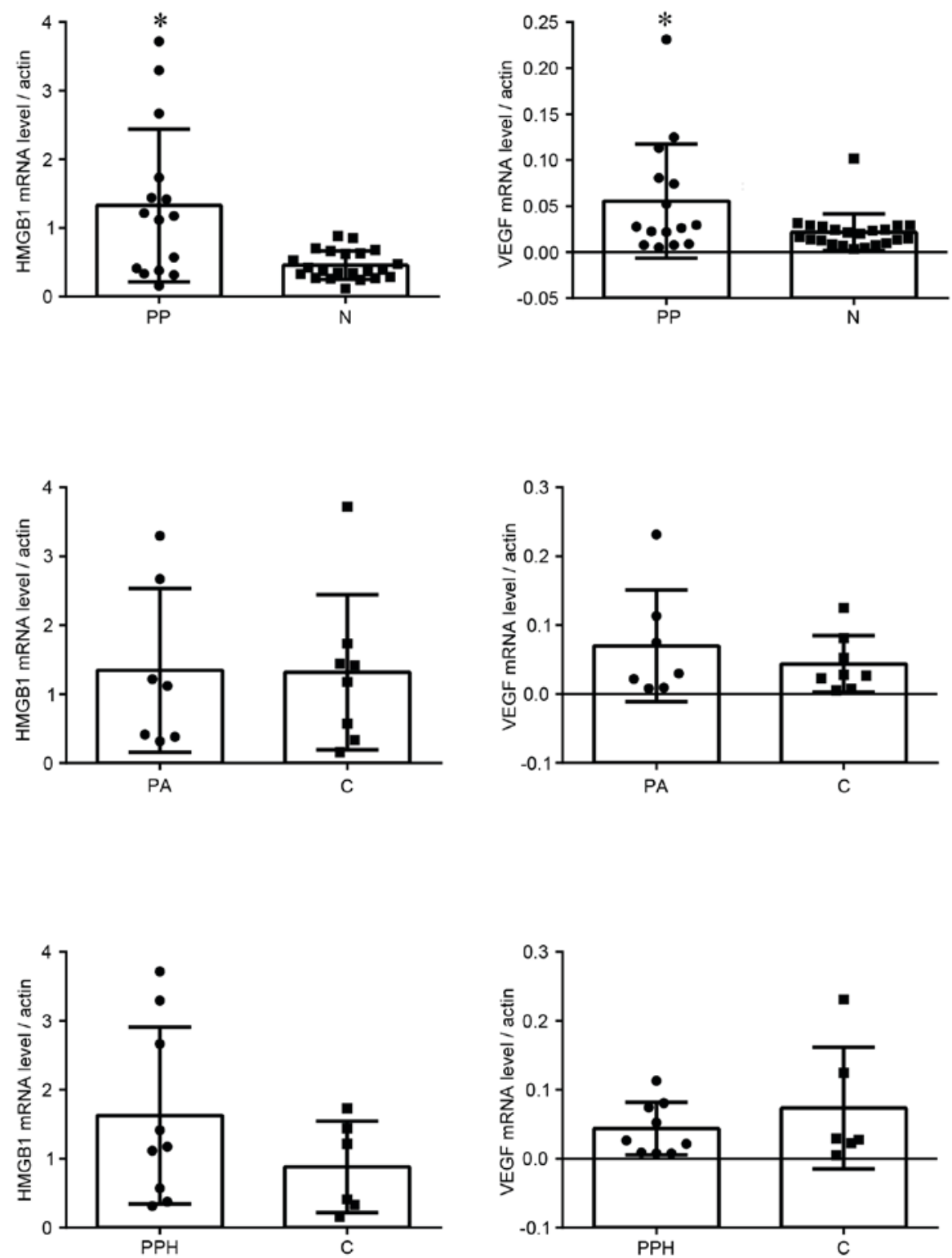

Figure 1. HMGB1 and VEGF mRNA expression levels, as detected by RT-qPCR. RT-qPCR revealed that the relative mRNA expression levels of HMGB1 and VEGF were increased in the PP group compared with in the $\mathrm{N}$ group. " $\mathrm{P}<0.05$ vs. the $\mathrm{N}$ group. The relative levels of HMGB1 and VEGF mRNA were not significantly different between groups with or without PA, or between groups with or without PPH. PP, placenta previa; N, normal; PA, placenta accreta; PPH, postpartum haemorrhage; C, control; HMGB1, high mobility group box protein 1; VEGF, vascular endothelial growth factor.

the placenta previa group was divided into the postpartum haemorrhage and control groups; no statistically significant difference was detected between these two groups (Fig. 4).

Histological and immunohistochemical examination of VEGF in placental tissue. VEGF was strongly and consistently expressed in trophoblasts and vascular endothelial cells covering the peripheral chorionic villi, predominantly in the cytoplasm of syncytiotrophoblasts and cytotrophoblasts, in both placenta previa and normal placental samples (Fig. 3B). According to the VEGF staining scores there were significant differences in VEGF expression in the villous trophoblasts and vascular endothelial cells of the terminal villi between the placenta previa and normal groups. As aforementioned, the placenta previa group was divided into placenta accreta and control groups; there were no significant differences in VEGF expression between these two groups. In addition, VEGF expression was not associated with postpartum haemorrhage (Fig. 4).

\section{Discussion}

Placenta previa is often associated with preterm delivery, reduced birth weight, a higher frequency of placental accreta and postpartum haemorrhage, and an increased likelihood of blood transfusion treatment. Considerable rates of maternal and fetal morbidity and mortality are associated with placenta previa; therefore, placenta previa is associated with a high demand for health care resources. A previous study regarding placenta previa focused on clinical epidemiology, ultrasonic prediction and vascular characteristics of the maternal-fetal interface, using digital technology (3). Research regarding the underlying molecular mechanisms of placenta previa is relatively rare. In recent years, studies into the molecular 


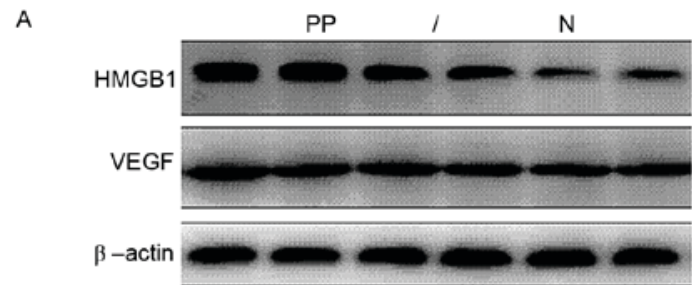

B
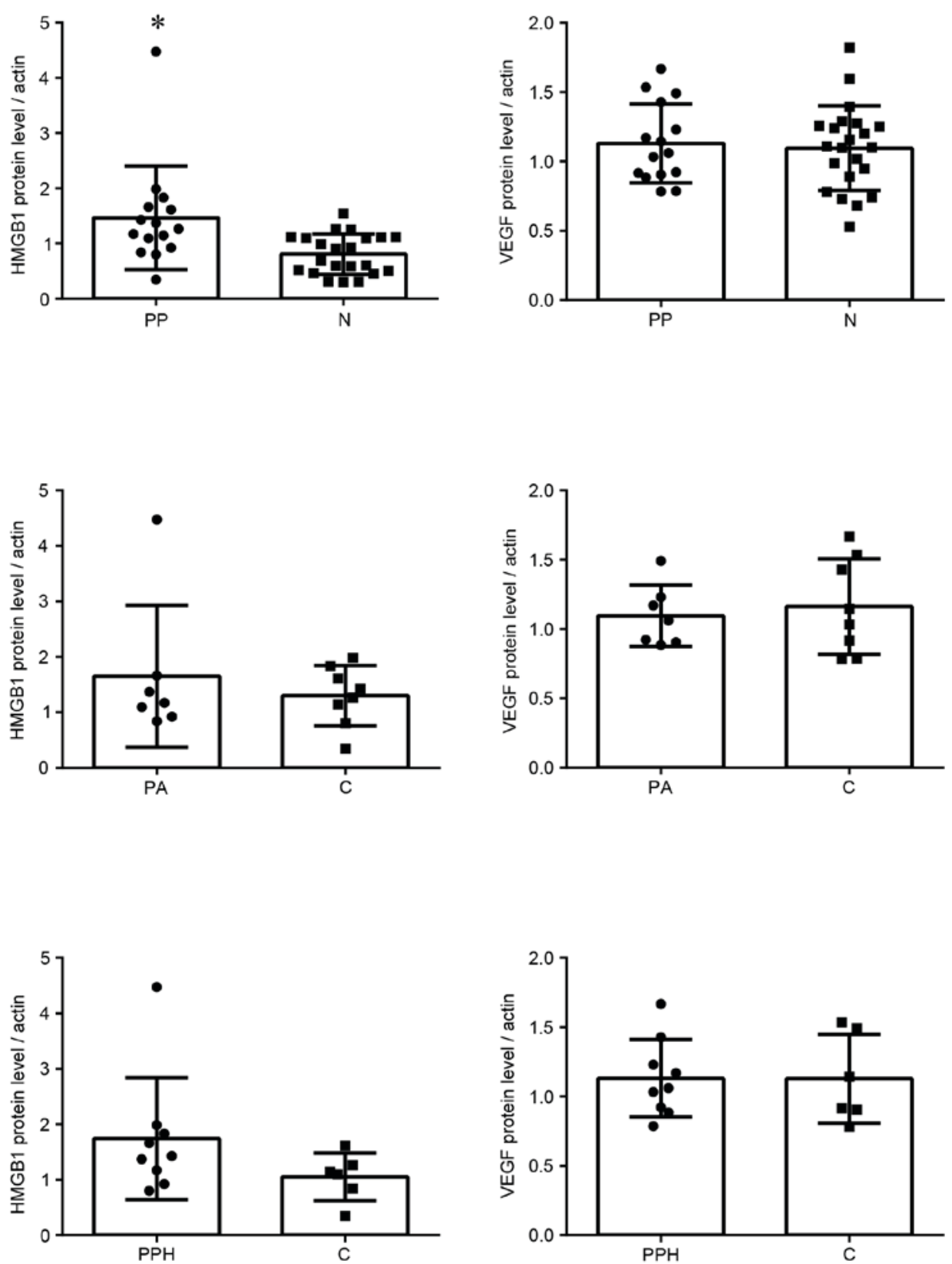

Figure 2. HMGB1 and VEGF protein expression levels, as detected by western blotting. (A) Western blot for HMGB1 and VEGF. $\beta$-actin was used as an internal control. (B) Western blot analysis indicated that HMGB1 protein expression was increased in the PP group compared with in the $\mathrm{N}$ group. * $\mathrm{P}<0.05$ vs. the $\mathrm{N}$ group. The protein expression levels of VEGF were not significantly different between the PP and N groups. HMGB1 and VEGF protein expression levels were not significantly different between groups with or without PA, or between groups with or without PPH. PP, placenta previa; N, normal; PA, placenta accreta; PPH, postpartum haemorrhage; C, control; HMGB1, high mobility group box protein 1; VEGF, vascular endothelial growth factor.

regulation of trophoblast cell infiltration and placental angiogenesis have garnered attention.
HMGB1 is constitutively and abundantly expressed in almost every eukaryotic cell type (15). HMGB1 is able to 


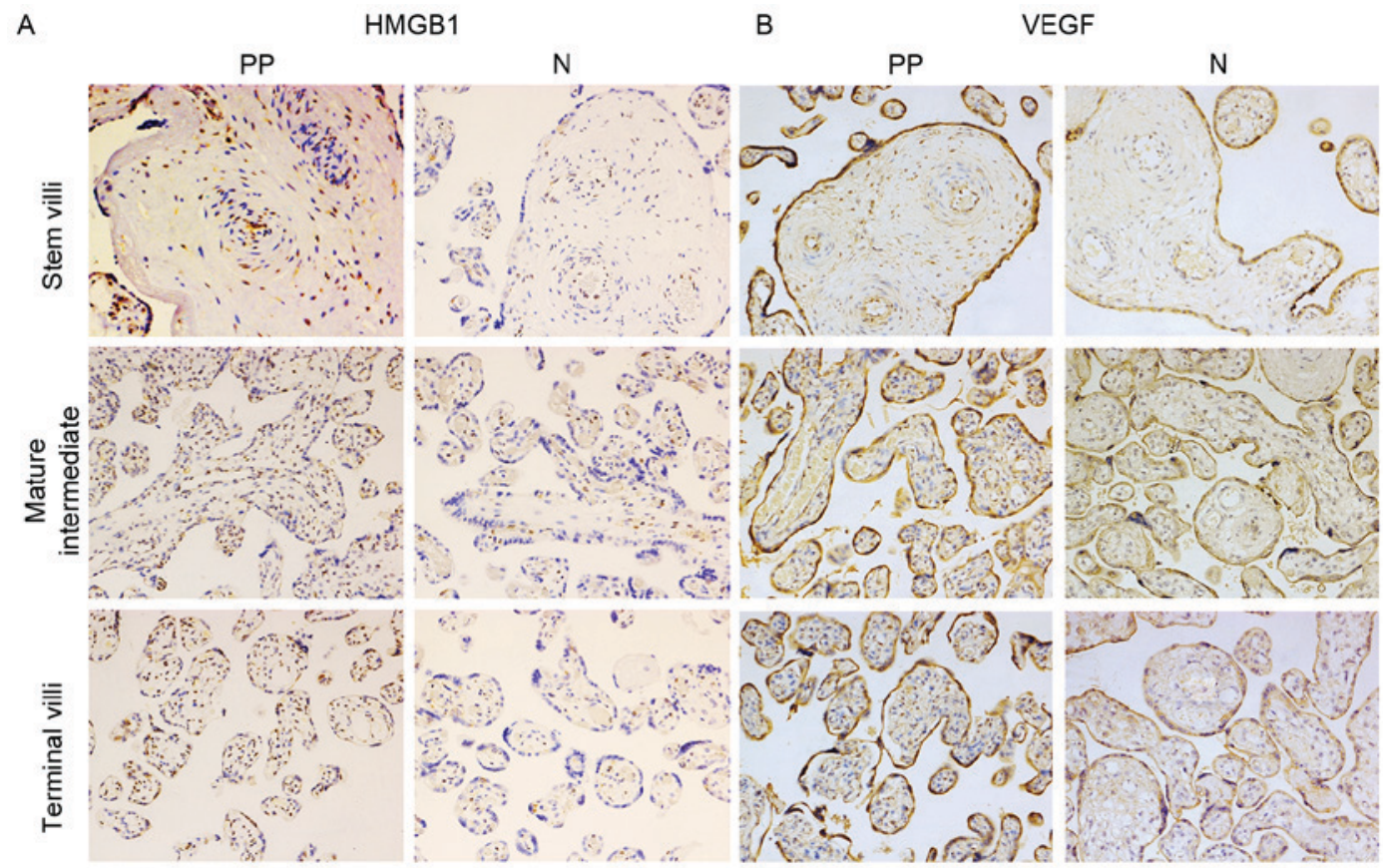

Figure 3. HMGB1 and VEGF protein expression was detected by immunohistochemistry. (A) HMGB1 protein was predominantly expressed in vascular endothelial cells and mesenchymal cells, and was increased in the placenta previa group. (B) VEGF protein was predominantly expressed in villous trophoblasts and vascular endothelial cells. Original magnification, x200. PP, placenta previa; N, normal group; HMGB1, high mobility group box protein 1; VEGF, vascular endothelial growth factor.

promote tumour cell migration (16) and has been hypothesised to be a potent cytokine mediating the late response to infection, injury and inflammation (17-20).

Since labour is an inflammatory-like process, HMGB1 may also be involved in embryogenesis and the process of pregnancy (21). Therefore, it may be hypothesised that there is a difference in the placental expression of HMGB1 between normal pregnancies and pregnancies complicated by intrauterine infection (22). Wang et al (10) investigated HMGB1 was expressed highly in preeclamptic placental tissue, which is a pregnancy-related complication characterised by poor placentation resulting in hypoxic placental conditions and an increased inflammatory response.

Based on the pathogenesis of inflammatory injury and angiogenesis of placenta previa, the present study hypothesised that HMGB1 may promote the occurrence of placenta previa. Therefore, the study aimed to determine the expression levels ofHMGB1 in placental tissues from women with or without placenta previa. The results confirmed that the mRNA expression levels of HMGB1 in the placenta were markedly higher in the placenta previa group compared with in the control group, as determined by RT-qPCR. In addition, the protein expression levels of HMGB1 were detected by western blotting; similarly, HMGB1 protein levels were increased in the placenta previa group. These findings suggested that HMGB1 may be associated with the emergence and progression of placenta previa.

PCR and western blotting were used to determine the mRNA and protein expression levels of HMGB1 in whole placental tissue samples. To determine if there were differences among the various cell types, immunohistochemistry was conducted. Understanding the location of HMGB1 expression may provide the basis for the selection of cell types in further cell function studies. As expected, in addition to strong nuclear HMGB1 expression in almost all cells in the studied placentas, an individual variation in cytoplasmic HMGB1 expression was detected in trophoblasts and vascular endothelial cells. Specifically, a higher cytoplasmic expression of HMGB1 was detected in trophoblasts and vascular endothelial cells from placenta previa placentas compared with in placentas from women with healthy pregnancies. The intracellular abundance of HMGB1 and its proinflammatory activities suggested that its release/secretion at tissue damage sites may serve an important role during inflammatory and immune responses (23). A previous study (24) demonstrated notable cytokine-like roles for extracellular HMGB1. Extracellular HMGB1 regulates cytokine expression and induces inflammatory cell recruitment. Furthermore, HMGB1 may stimulate migration of adherent cells, such as fibroblasts and smooth muscle cells (25). Therefore, extracellular HMGB1 may be regarded as a tissue injury signal and an inflammatory mediator. These findings suggested that, as a proinflammatory cytokine, HMGB1 may be secreted to the extracellular matrix, participate in the inflammatory response and prompt the progression of placenta previa.

The immunohistochemistry results demonstrated that HMGB1 expression was markedly increased in villous vascular endothelial cells and mesenchymal cells in the placenta previa group. These results indicated that HMGB1 may have a relevant role in angiogenesis. According to the theory of embryonic development, villous vascular endothelial cells and mesenchymal cells originate from the mesenchymal cells of embryonic mesoderm, and then differentiate into vascular and connective tissues, alongside development of the placental villus. Therefore, protein expression is always relatively consistent in these cells. This was confirmed in the 

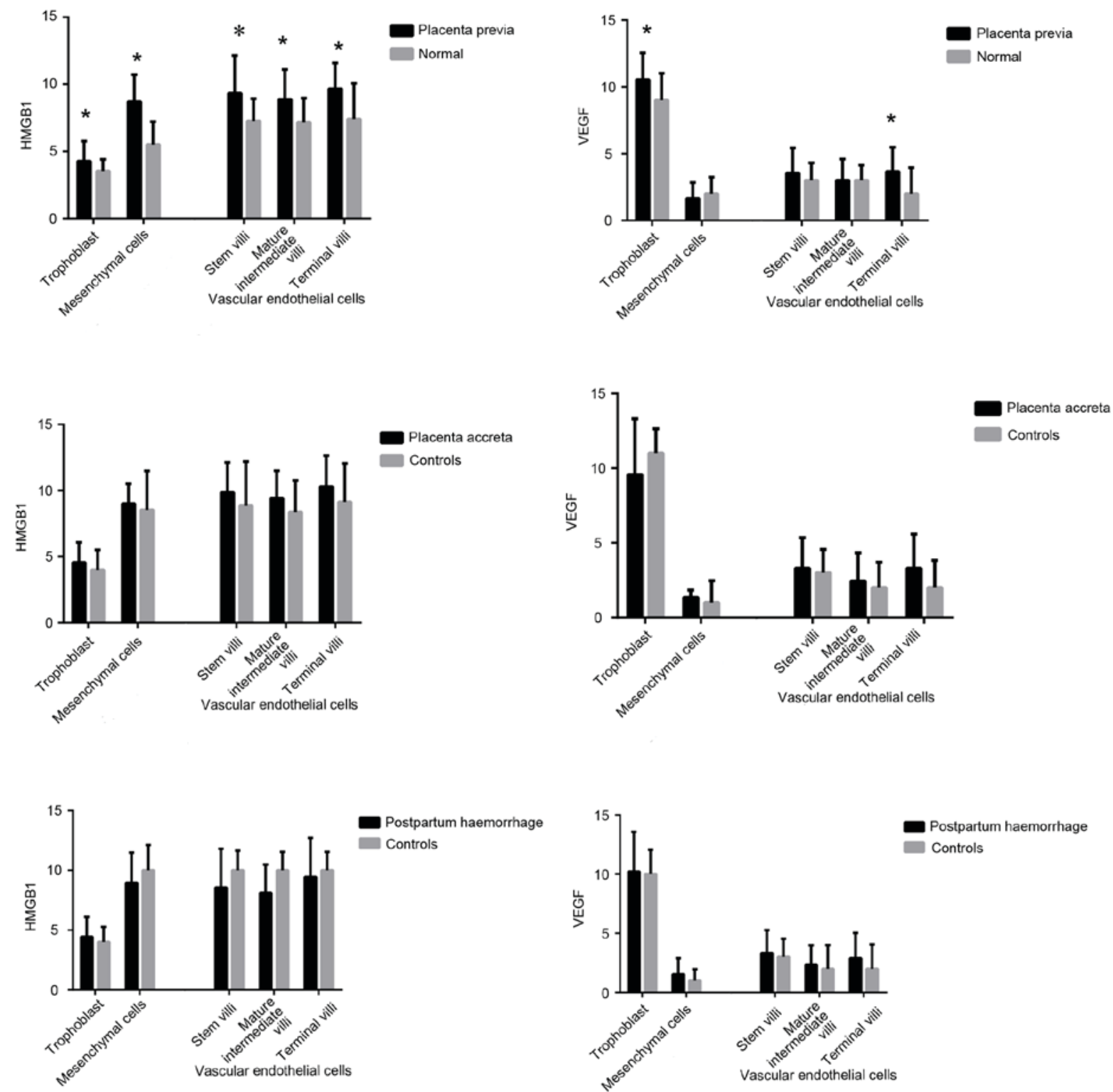

Figure 4. Staining scores of immunohistochemistry results. HMGB1 staining was increased in vascular endothelial cells, mesenchymal cells and trophoblast cells in the placenta previa groups compared with in the normal group. VEGF staining was increased in villous trophoblasts and vascular endothelial cells of the terminal villi in the placenta previa group compared with in the normal group. " $\mathrm{P}<0.05$ vs. the normal group. No statistically significant differences in HMGB1 and VEGF staining were detected between the placenta accreta group and the control group, or between the postpartum haemorrhage group and the control group.

results of the present study, which demonstrated that HMGB1 expression was higher in villous vascular endothelial cells and mesenchymal cells than in trophoblast, whereas the opposite was observed for expression of VEGF. Due to the importance of neovascularization at the site of injured tissue, where blood flow restoration is often required for the initiation of an immune response to pathogens and for subsequent successful wound repair (26), the capacity of extracellular HMGB1 to exert a potent angiogenic activity strengthens the importance of HMGB1 as a cytokine. The immunohistochemistry results may also indicate that HMGB1 exerts a prominent role in numerous processes of specific interest for the placenta, such as angiogenesis, in addition to its potent proinflammatory capacities.

Given its cytokine features, a previous study investigated the capacity of HMGB1 to modulate the various steps of angiogenesis in vitro, and examined its proangiogenic activity in vivo (27). In the ischemic muscle of diabetic mice, HMGB1 administration restored blood flow recovery and capillary density; this process was associated with the increased expression of VEGF, whereas HMGB1-induced angiogenesis was significantly reduced following suppression of VEGF activity (28). In addition, patients with non-small cell lung cancer have been reported to possess a higher serum concentration of HMGB1 and VEGF $(29,30)$. In oesophageal squamous cell carcinoma, HMGB1 was highly expressed and affected the prognosis of patients via regulation of VEGF-C expression, which promoted lymph angiogenesis and lymph node metastasis (30). In a previous study regarding preeclampsia, it was suggested that the tendency towards a higher expression of HMGB1 in preeclamptic placentas may be a result of hypoxia, and may be considered a compensatory mechanism for the 
placenta to attempt to increase vascularization (31). Due to the findings of these previous studies and the similarities between placental angiogenesis and tumour growth, HMGB1 may be considered to serve an important role in placentation; however, this remains to be investigated.

Although the cause of placenta previa remains unknown, growing evidence has suggested that an imbalance between pro- and anti-angiogenic factors may have a fundamental role in its pathogenesis. Therefore, it was hypothesised that HMGB1 may regulate placental angiogenesis in placenta previa through the expression of VEGF. In the present study, the expression levels of VEGF were detected in placental samples from women with placenta previa by RT-qPCR and western blot analysis. The results indicated that the mRNA expression levels of VEGF were significantly higher in placental tissue from the placenta previa group, whereas there was no significant difference in the protein expression of VEGF between the placenta previa and normal groups. This may be due to the limited sample numbers in the present study. Subsequently, immunohistochemical staining was used to locate VEGF protein expression. A strong cytoplasmic VEGF expression was detected in villous trophoblasts and vascular endothelial cells, particularly in trophoblasts. Furthermore, VEGF expression was significantly increased in the placenta previa group. This finding is in accordance with the theory that VEGF regulates trophoblast invasion through autocrine modes of action, and promotes placental vascularization in a paracrine manner (32).

Placenta accreta is associated with a highly regulated inflammatory-like response and vascularization. Size and spatial organization of the placenta-increta vascular architecture at the placental-maternal interface differs from normal and may partially explain the severe haemorrhage observed during delivery of placenta-increta (7). Since HMGB1 is a potent inflammatory and proangiogenic cytokine, the present study aimed to investigate placental HMGB1 expression in relation to placenta accreta and postpartum haemorrhage. The placenta previa group was divided into placenta accrete and postpartum haemorrhage groups. However, there were no differences in the expression of HMGB1 and VEGF in the placenta accreta/postpartum haemorrhage groups compared with in the control placenta previa group. These results suggested that placental angiogenesis may have a role in placenta increta, where the placenta attaches itself even more deeply into the muscle wall of uterus, rather than in placenta accreta. However, these negative findings may be associated with the limited sample numbers.

In conclusion, the present study demonstrated that HMGB1 may participate in the progression of placenta previa, not only through its role as a proinflammatory cytokine but also as a proangiogenic cytokine. However, the precise role of HMGB1 in placenta previa and the underlying molecular mechanisms remain to be elucidated. In addition, the signalling pathways through which HMGB1 mediates VEGF expression in placenta previa require further study.

\section{Acknowledgements}

The present study was funded by the Health and Family Planning commission of Pudong District, Shanghai (grant no. PW2012D-10); the Key Program of Health and Family Planning commission of Shanghai (grant no. 20124037); the Shanghai Hospital Development Center Grant (grant no. SHDC12012116); the Natural Science Foundation of Shanghai (grant no. 13ZR1432900); the Shanghai Science and Technology Committee (grant no. 134119a0800); the Industrial, Teaching and Research Cooperative Program in Medical Field, Science and Technology Commission of Shanghai Municipality (grant no. 13DZ1931002); and the National Natural Science Foundation of China (NSFC) (grant no. 81200443).

\section{References}

1. Royal College of Obstetricians and Gynaecologists (RCOG): Placenta Praevia, Placenta Praevia Accreta and Vasa Praevia: Diagnosis and Management. RCOG Green-top Guideline No. 27. https://www.rcog.org.uk/globalassets/documents/guidelines/gtg_27.pdf. Accessed January, 2011.

2. Oya A, Nakai A, Miyake H, Kawabata I and Takeshita T: Risk factors for peripartum blood transfusion in women with placenta previa: A retrospective analysis. J Nippon Med Sch 75: 146-151, 2008.

3. Publications Committee, Society for Maternal-Fetal Medicine and Belfort MA: Placenta accreta. Am J Obstet Gynecol 203: 430-439, 2010.

4. Usta IM, Hobeika EM, Musa AA, Gabriel GE and Nassar AH: Placenta previa-accreta: Risk factors and complications. Am J Obstet Gynecol 193: 1045-1049, 2005

5. Gielchinsky Y, Rojansky N, Fasouliotis SJ and Ezra Y: Placenta accreta-summary of 10 years: A survey of 310 cases. Placenta 23: 210-214, 2002.

6. Ananth CV, Smulian JC and Vintzileos AM: The association of placenta previa with history of cesarean delivery and abortion: A meta analysis. Am J Obstet Gynecol 177: 1071-1078, 1997.

7. Chantraine F, Blacher S, Berndt S, Palacios-Jaraquemada J, Sarioglu N, Nisolle M, Braun T, Munaut C and Foidart JM: Abnormal vascular architecture at the placental-maternal interface in placenta increta. Am J Obstet Gynecol 207: 188.e1-189. e1, 2012 .

8. Knofler M: Critical growth factors and signalling pathways controlling human trophoblast invasion. Int J Dev Biol 54: 269-280, 2010.

9. Abe A, Kuwata T, Yamauchi C, Higuchi Y and Ochiai A: High mobility group box1 (HMGB1) released from cancer cells induces the expression of pro-inflammatory cytokines in peritoneal fibroblasts. Pathol Int 64: 267-275, 2014.

10. Wang B, Koga K, Osuga Y, Hirata T, Saito A, Yoshino O, Hirota Y, Harada M, Takemura Y, Fujii T, et al: High mobility group box 1 (HMGB1) levels in the placenta and in serum in preeclampsia. Am J Reprod Immunol 66: 143-148, 2011.

11. Knofler M: Critical growth factors and signalling pathways controlling human trophoblast invasion. Int J Dev Biol 54: 269-280, 2010.

12. Livak KJ and Schmittgen TD: Analysis of relative gene expression data using real-time quantitative PCR and the 2(-Delta Delta C(T)) method. Methods 25: 402-408, 2001.

13. Siu MK, Chan HY, Kong DS, Wong ES, Wong OG, Ngan HY, Tam KF, Zhang H, Li Z, Chan QK, et al: p21-activated kinase 4 regulates ovarian cancer cell proliferation, migration, and invasion and contributes to poor prognosis in patients. Proc Natl Acad Sci USA 107: 18622-18627, 2010.

14. Lu W, Xia YH, Qu JJ, He YY, Li BL, Lu C, Luo X and Wan XP: p21-activated kinase 4 regulation of endometrial cancer cell migration and invasion involves the ERK1/2 pathway mediated MMP-2 secretion. Neoplasma 60: 493-503, 2013.

15. Vitali R, Stronati L, Negroni A, Di Nardo G, Pierdomenico M, del Giudice E, Rossi P and Cucchiara S: Fecal HMGB1 is a novel marker of intestinal mucosal inflammation in pediatric inflammatory bowel disease. Am J Gastroenterol 106: 2029-2040, 2011.

16. Nehil M, Paquette J, Tokuyasu T and McCormick F: High mobility group box 1 promotes tumor cell migration through epigenetic silencing of semaphorin 3A. Oncogene 33: 5151-5162, 2014. 
17. Fang P, Pan HC, Lin SL, Zhang WQ, Rauvala H, Schachner M and Shen YQ: HMGB1 contributes to regeneration after spinal cord injury in adult zebrafish. Mol Neurobiol 49: 472-483, 2014.

18. Palone F, Vitali R, Cucchiara S, Pierdomenico M, Negroni A Aloi M, Nuti F, Felice C, Armuzzi A and Stronati L: Role of HMGB1 as a suitable biomarker of subclinical intestinal inflammation and mucosal healing in patients with inflammatory bowel disease. Inflamm Bowel Dis 20: 1448-1457, 2014.

19. Yang PS, Kim DH, Lee YJ, Lee SE, Kang WJ, Chang HJ and Shin JS: Glycyrrhizin, inhibitor of high mobility group box-1, attenuates monocrotaline-induced pulmonary hypertension and vascular remodeling in rats. Respir Res 15: 148, 2014

20. Campana L, Santarella F, Esposito A, Maugeri N, Rigamonti E, Monno A, Canu T, Del Maschio A, Bianchi ME, Manfredi AA, et al: Leukocyte HMGB1 is required for vessel remodeling in regenerating muscles. J Immunol 192: 5257-5264, 2014.

21. Bhutada S, Basak T, Savardekar L, Katkam RR, Jadhav G, Metkari SM, Chaudhari UK, Kumari D, Kholkute SD, Sengupta S, et al: High mobility group box 1 (HMGB1) protein in human uterine fluid and its relevance in implantation. Hum Reprod 29: 763-780, 2014.

22. Girard S, Heazell AE, Derricott H, Allan SM, Sibley CP, Abrahams VM and Jones RL: Circulating cytokines and alarmins associated with placental inflammation in high-risk pregnancies. Am J Reprod Immunol 72: 422-434, 2014.

23. Mitola S, Belleri M, Urbinati C, Coltrini D, Sparatore B, Pedrazzi M, Melloni E and Presta M: Cutting edge: Extracellular high mobility group box-1 protein is a proangiogenic cytokine. J Immunol 176: 12-15, 2006.

24. Lee SA, Kwak MS, Kim S and Shin JS: The role of high mobility group box 1 in innate immunity. Yonsei Med J 55: 1165-1176, 2014.
25. Degryse B, Bonaldi T, Scaffidi P, Muller S, Resnati M, Sanvito F, Arrigoni G and Bianchi ME: The high mobility group (HMG) boxes of the nuclear protein HMG1 induce chemotaxis and cytoskeleton reorganization in rat smooth muscle cells. J Cell Biol 152: 1197-1206, 2001.

26. Frantz S, Vincent KA, Feron $\mathrm{O}$ and Kelly RA: Innate immunity and angiogenesis. Circ Res 96: 15-26, 2005.

27. Schlueter C, Weber H, Meyer B, Rogalla P, Roser K, Hauke S and Bullerdiek J: Angiogenetic signaling through hypoxia: HMGB1: An angiogenetic switch molecule. Am J Pathol 166: 1259-1263, 2005.

28. Biscetti F, Straface G, De Cristofaro R, Lancellotti S, Rizzo P, Arena V, Stigliano E, Pecorini G, Egashira K, De Angelis G, et al: High-mobility group box-1 protein promotes angiogenesis after peripheral ischemia in diabetic mice through a VEGF-dependent mechanism. Diabetes 59: 1496-1505, 2010.

29. Naumnik W, Nilklinska W, Ossolinska M and Chyczewska E: Serum levels of HMGB1, survivin, and VEGF in patients with advanced non-small cell lung cancer during chemotherapy. Folia Histochem Cytobiol 47: 703-709, 2009.

30. Chuangui C, Peng T and Zhentao Y: The expression of high mobility group box 1 is associated with lymph node metastasis and poor prognosis in esophageal squamous cell carcinoma. Pathol Oncol Res 18: 1021-1027, 2012.

31. Holmlund U, Wahamaa $\mathrm{H}$, Bachmayer $\mathrm{N}$, Bremme $\mathrm{K}$, Sverremark-Ekstrom E and Palmblad K: The novel inflammatory cytokine high mobility group box protein 1 (HMGB1) is expressed by human term placenta. Immunology 122: 430-437, 2007.

32. Tseng JJ, Chou MM, Hsieh YT, Wen MC, Ho ES and Hsu SL: Differential expression of vascular endothelial growth factor, placenta growth factor and their receptors in placentae from pregnancies complicated by placentaaccreta. Placenta 27: 70-78, 2006. 